\title{
Performance Evaluation of Vehicle-to-Vehicle Communication for Cooperative Collision Avoidance at Urban Intersections
}

\author{
Ibrahim Rashdan, Martin Schmidhammer, Fabian de Ponte Müller and Stephan Sand, Senior Member, IEEE \\ German Aerospace Center (DLR) \\ Institute of Communications and Navigation, 82234 Oberpfaffenhofen, Germany \\ \{Ibrahim.Rashdan | Martin.Schmidhammer | Fabian.PonteMueller | Stephan.Sand\}@dlr.de
}

\begin{abstract}
Vehicle-to-Vehicle (V2V) communication can significantly enhance the performance of collision avoidance systems through periodically exchanging information between vehicles. At urban intersections, the effect of shadowing caused by buildings has a severe influence on the communication performance. In addition, an increased traffic density near an intersection creates a high level of interference, which can lead to a communication performance degradation. In this paper, we evaluate the performance of IEEE 802.11p based V2V communication for cooperative collision avoidance at urban intersections. We focus on the impact of shadowing from buildings and of traffic density on the communication performance. For this purpose, we investigate the effect of both the data rate and transmission power in different scenarios. The simulation results show that transmission power and data rate can be tailored to increase the reliability of the communication for the collision avoidance system. Simulation results also show that buildings at intersections reduce the interference from vehicles in other road sections. In addition, our simulation results reveal more insight on the main cause of packet loss near intersections. They show that loss due to packet collisions is the dominant reason, which can be reduced by increasing the data rate.
\end{abstract}

Index Terms-5.9 GHz, Cooperative, V2V, Shadowing, Collision avoidance, Intersection

\section{INTRODUCTION}

With the increasing number of vehicles, an increasing of number of crashes has been reported over the last years, particularly at urban intersections. According to the federal statistical office of Germany, 75,239 intersection-related crashes occurred in Germany in 2015 [1]. Current collision avoidance systems are based on in-vehicle sensors, like radar [2], stereo camera, laser [3] or fusion of sensors for vehicle detection [4]. However, in-vehicle sensors suffer from limitations e.g. sensitivity to weather conditions and they require line-of-sight (LoS) for object detection. Yet, in most intersection crash scenarios, the vehicles are hidden behind buildings in different road sections until the last second before the crash. In order to overcome the limitations of the conventional sensor-based collision avoidance system and to enhance safety, wireless communication is used to enable vehicles with 360-degree awareness about the neighboring vehicles and critical situations. Communication can significantly enhance the performance of collision avoidance systems by the periodic exchange of information between vehicles and between vehicles and infrastructure. The system cooperatively predicts collisions and alerts the driver. Then, it overrides the driver control to avoid or mitigate the predicted collision. Hence, it reduces the number of collisions on the roads or mitigates the severity when these collisions are unavoidable. The cooperative collision avoidance systems will be based on the IEEE $802.11 \mathrm{p}$ standard. The European profile of IEEE $802.11 \mathrm{p}$ is known as ETSI ITS-G5 and it operates in the $5.9 \mathrm{GHz}$ frequency band. Due to the nature of the intersection topologies, providing reliable communication at intersections, more specifically, at urban intersections, is very challenging. Stationary and mobile obstacles at the intersection may obstruct the link between two vehicles completely or partially. The LoS being blocked by buildings significantly affects the signal quality at the receiver side which leads to packet losses or can cause a communication breakdown. Moreover, the worst case on the channel happens at an urban intersection where there is a high density of vehicles operating in the vicinity of the intersection. High density leads to high interference and packet collisions, which result in low communication performance.

Based on both simulations and measurements, the impact of diverse environmental conditions on the performance of $\mathrm{V} 2 \mathrm{~V}$ communication at road intersections is investigated in multiple recent research studies. For urban environments, the quality of received signals at intersections is strongly affected by nearby buildings and geometry aspects, as analyzed in [6]. Mahler et al. [7] show that the effect of shadowing caused by buildings is the most severe influence on the communication performance. The work in [8] addresses the impact of radio shadowing, which is identified as the cause of strongly time-variant signals in vehicular networks. The effect of channel load is also subject of investigation in the simulation-based study in [9]. In [10], the authors study the effect of interference on the safetycritical communication on a highway. Adapting the update rate based on the environment in order to improve the reliability of vehicle safety applications is studied in [11]. The analysis of the cumulative effect of shadowing at intersections is the motivation for [12], which is also the closest work to ours. However, in our work, we particularly address the cooperative collision avoidance at urban intersection and study the effect of traffic density, transmission power, data rate, and the in- 
tersection topology on the performance of the communication system at an intersection. In addition, we trace the cause of packet losses by distinguishing between bit error and packet collisions induced losses. We believe that this is the first time that such a parametric study and performance evaluation has been conducted. Moreover, existing studies have only dealt with low and moderate traffic densities, whereas in our study, the case of high traffic density where the channel is saturated is studied.

\section{Communication for Collision Avoidance}

From communication and operational point of view, we can distinguish between three operational phases. The three phases are: detection, pre-crash and post-crash phases. Each phase will impose different communication requirements and communication types. In this paper we focus on the detection phase where all vehicles periodically broadcast beacon messages which are called Cooperative Awareness Messages (CAMs). According to the standard [13], vehicles exchange their state information like position, speed, and heading. The purpose of CAMs is to create awareness of the own vehicle by broadcasting with single-hop communication and at an update rate of $10 \mathrm{~Hz}$. At the receiving node, the information from all neighbors is stored and used to build a Local Dynamic Map (LDM) to allow cooperative awareness and to calculate the collision probabilities with all vehicles. When a collision is detected, all vehicles that are involved in the collision enter the pre-crash phase for maneuver negotiation in order to avoid or mitigate the collision. Note that, detection methods and precrash phase are out of the scope of this work.

\section{A. Communication Impairments at Intersection}

This study focuses on the effects of interference and radio shadowing as the two key challenges of $\mathrm{V} 2 \mathrm{~V}$ communication at urban intersections. The problem of channel interference arises from the simultaneous data transmission of at least two nodes in the same channel. Thus, it is strongly related to the coordination of the access to the shared wireless channel. For IEEE 802.11p the Media Access Control (MAC) layer employs the fully decentralized Carrier Sense Multiple Access with Collision Avoidance (CSMA/CA) protocol. This contention- and random-based MAC protocol neither guarantees deterministic channel access nor avoids packet collisions caused by simultaneous channel usage at high traffic densities. In addition to channel congestion, packet collisions can occur due to hidden terminal problem. A hidden terminal is defined as another communicating node being in range of a dedicated receiver but out of range to the corresponding transmitter. Amongst others, the occurrence of such nodes can be caused by signal blockage, which refers to the effect of radio shadowing. In urban environments, shadowing is mainly caused by solid buildings or mobile objects such as trucks or cars blocking the LoS signal propagation for a short time , e.g. a few milli seconds.

\section{B. Performance Metric}

The traditional packet delivery ratio (PDR) and end-to-end (E2E) delay provide the average value over time but do not consider any correlation between the subsequent transmission and reception. To illustrate this point, let us consider the following example: $99 \%$ of PDR, i.e. 100 packets out of 10,000 are lost. This does not provide information on whether the packet loss has a uniform distribution or whether all packets are lost in one burst of errors. Clearly, the later one is critical for safety. Since we want to analyze in this paper the performance of a communication system for collision avoidance, we use the Update Delay (UD), which is a suitable performance metric.

The UD is defined as the time elapsed between two consecutive successfully received CAMs from a specific transmitter at a specific receiver. The UD values can be one or multiple transmission intervals depending on the number of consecutive lost CAMs. Losing CAM message from another vehicle will decrease the awareness quality about the position and speed of that vehicle. With each lost packet the UD increases. With decreasing awareness quality due consecutive lost CAMs, hazardous and unsafe situations may occur.

To visualize the UD, we chose the complementary cumulative distribution function (CCDF), which corresponds to the probability that the UD is larger than a time t. The CCDF is a step function since the periodic transmissions of CAM messages are scheduled according to a chosen update rate. For a specific application one can define the achieved reliability that a UD value is not exceeded.

\section{Simulation}

In order to evaluate the performance of the ITS-G5 based $\mathrm{V} 2 \mathrm{~V}$ communication for cooperative collision avoidance, we conducted simulations at urban intersection scenarios. Our main aim is to study the effect of shadowing and interference on the communication performance during the detection phase. For this purpose, we perform simulations in open and closed intersections and with different combinations of traffic density, transmission power, and data rate. We refer to an intersection as closed when the four corners of the intersection are occupied by buildings. Similarly, the intersection is called open when the four buildings are absent.

\section{A. Simulation environment and setup}

The simulation environment consists of SUMO and OM$\mathrm{NeT}++$ as traffic and network simulator respectively. SUMO is a microscopic, open source road traffic simulator used to handle the mobility of the vehicles. OMNeT++ is a discrete event simulator used for modeling communication networks. To couple SUMO with OMNeT++, the VEINS framework was used. We consider vehicles approaching a four-way intersection from all directions. The intersection is controlled by a traffic light and each road section is $1 \mathrm{~km}$ long and consists of 6 lanes, 3 lanes in each direction. Fig. 1 shows the scenario of a closed intersection layout.

In order to get a more realistic vehicle distribution, we add a warm-up time of 200 seconds in the beginning of each simulation without any communications, i.e. no packets are sent. The main simulation parameters are given in Table I and Table II. 


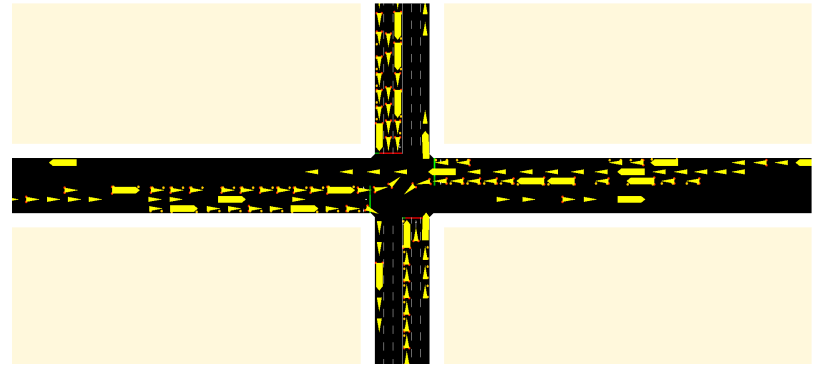

Fig. 1: Closed intersection layout.

TABLE I: Simulation parameters

\begin{tabular}{ll}
\hline Parameter & Value \\
\hline Max. velocity & $60 \mathrm{~km} \mathrm{~h}^{-1}$ \\
\hline Low traffic density & $15 \mathrm{veh} / \mathrm{km} /$ lane \\
\hline High traffic density & $110 \mathrm{veh} / \mathrm{km} /$ lane \\
\hline Transmission power & $13,23 \mathrm{dBm}$ \\
\hline Receiver sensitivity & $-85,-82,-73 \mathrm{dBm}$ \\
\hline Data rate & $3,6,18 \mathrm{Mbps}$ \\
\hline CAM update rate & $10 \mathrm{~Hz}$ \\
\hline CAM size & $338 \mathrm{Byte}$ \\
\hline
\end{tabular}

The main goal is to increase mutual awareness between vehicles that are approaching the intersection. As shown in [14], it is highly unlikely that a collision occurs between vehicles that have large difference in distance to intersection. This is because they will arrive at the intersection at different times. For the aforementioned reason, the results for each simulation scenario are drawn from the UDs of the received CAMs within a region of interest (RoI) in which both the transmitter and the receiver are within $200 \mathrm{~m}$ from the center of the intersection and thus the edge effect is completely avoided.

\section{B. Propagation model}

In the simulations, we adopted a computationally inexpensive empirical obstacle model from [15] that can accurately capture shadowing effect in vehicular ad hoc networks (VANETs). In this model, the additional attenuation due to an obstacle in the LoS between the transmitter and the receiver is given by $L_{o}$ in $\mathrm{dB}$.

$$
L_{o}=\beta n+\gamma d_{m}
$$

where $n$ is the number of times the boarder of the obstacle intersect by the LoS and $d_{m}$ is the length of the obstacle

TABLE II: Data rate and receiver sensitivity for modulation and coding schemes.

\begin{tabular}{llll}
\hline Modulation & BPSK & QPSK & 16-QAM \\
\hline Coding rate & $1 / 2$ & $1 / 2$ & $3 / 4$ \\
\hline Data rate $[\mathrm{Mbps}]$ & 3 & 6 & 12 \\
\hline Receiver sensitivity $[\mathrm{dBm}]$ & -85 & -82 & -73 \\
\hline
\end{tabular}

TABLE III: Propagation model parameters

\begin{tabular}{ll}
\hline Parameter & Value \\
\hline$\alpha$ & 2 \\
\hline$\beta$ & $9 \mathrm{~dB} /$ wall \\
\hline$\gamma$ & $0.4 \mathrm{~dB} / \mathrm{m}$ \\
\hline
\end{tabular}

intersection. $\beta$ is a calibration factor that is given in $\mathrm{dB}$ per wall and it represents the attenuation due to the exterior wall of a building. The internal structure of a building is roughly approximated using another calibration factor $\gamma$ in $\mathrm{dB}$ per meter. The shadowing model in (1) is then combined with a generic free space path loss model

$$
P_{r}=P_{t}+10 \lg \left(\frac{G_{t} G_{r} \lambda^{2}}{16 \pi^{2} d^{\alpha}}\right)-\beta n-\gamma d_{m},
$$

where $\lambda$ is the wavelength, $d$ is the distance between transmitter and receiver, $P_{r}$ and $P_{t}$ are the received power and the transmitted power in $\mathrm{dBm}$ respectively, $G_{t}$ and $G_{r}$ are the antenna gains and $\alpha$ is the path loss exponent. The assumed values for the calibration factors and the path loss exponent are given in Table III and are adopted from [15].

\section{RESUlts}

In this section, we evaluate the effect of transmission power, traffic density, type of intersection and data rate on the UD. All UD curves consider all communication links between all vehicles within the RoI. In the CCDF of the UD curves, the time is shown on the $\mathrm{x}$-axis, the probability that the UD is greater than a certain $\mathrm{x}$-value is assigned to the $\mathrm{y}$-axis.

\section{A. The effect of traffic density and transmission power}

The first analysis aims at comparing the effect of traffic density and transmission power on the awareness quality of vehicles. In Fig. 2, the UD curves are shown up to 1 s for the open intersection in different traffic densities and transmission power. Overall, it can be seen that increasing traffic density has a negative effect on the awareness quality, since the wireless communication resources are limited and have to be shared amongst more vehicles. This is confirmed by Fig. 2, as the probability of losing more than ten consecutive CAMs from a certain receiver (equivalent to an UD equal to $1 \mathrm{~s}$ ) is increased by factor 117 and factor 38 when the transmission power is $23 \mathrm{dBm}$ and $13 \mathrm{dBm}$ respectively. Note that the actual car traffic density only increased by factor of approximately 8 .

This additional degradation in performance is due to an increased interference. Similarly, a higher transmission power will increase the communication range and hence will increase the interference. As a result, more packet collisions occur.

\section{B. The effect of buildings}

In contrast to open intersections, communication at closed intersections suffers from LoS-obstraction due to buildings. the decreased received signal power has a negative impact on the awareness range. However, the four buildings at the intersection have a beneficial side-effect on the UD, since they decrease interference from vehicles located at other road 


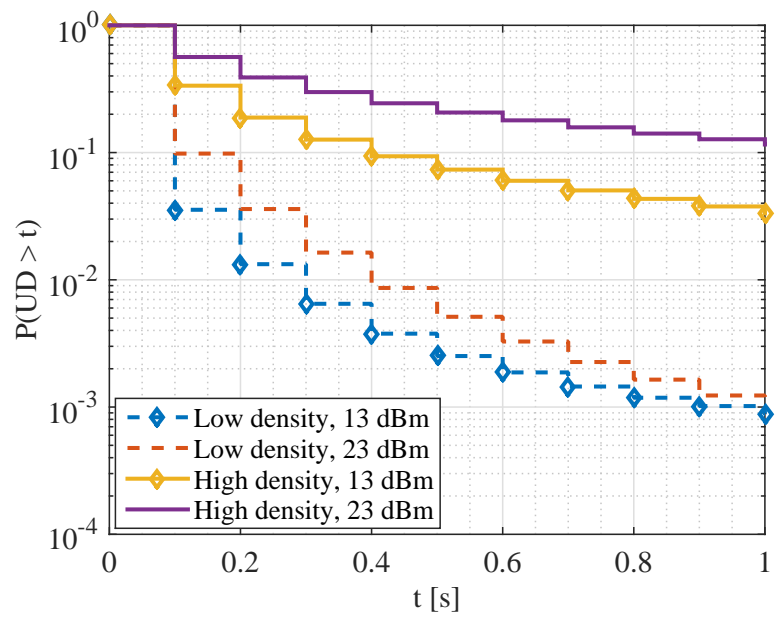

Fig. 2: CCDF of the UD in different traffic densities and transmission power at an open intersection and a data rate of 6 Mbps.

sections. This effect of surrounding buildings of an urban intersection on the awareness quality of vehicles can be seen by comparing Fig. 3 with Fig. 2, especially for the high density scenario.

\section{The effect of data rate}

Basically, a higher order modulation scheme provides higher data rates and requires higher received signal strength to achieve the same performance of lower order modulation schemes. Furthermore, higher order modulation schemes are not as robust in the presence of noise as lower order modulation schemes. Based on this fact, we expected to achieve better performance by lowering the modulation scheme and the data rate. However, our results in Fig. 4 contradict our expectations. Interestingly, the results show that the UD performance is better with higher data rate.

To explain these results, the signal-to-noise ratio (SNR) of each received message is calculated. The SNR is the ratio of the received signal power, $P_{r}$, to the in-band noise power, $P_{n}$. Each type of modulation tends to behave in different way in the presence of noise. More specifically, higher order modulation schemes that provide higher data rates are less robust in presence of noise than the lower order modulation. Therefore, the higher the order of modulation the higher the receiver sensitivity threshold and the SNR that is required in order to achieve a specific level of bit error rate.

Our simulations show that the medium between the transmitter and the receiver is good enough and results in high SNR for the three modulation schemes. $100 \%$ of the received messages have an SNR higher than 25,28 , and $37 \mathrm{~dB}$ when using BPSK, QPSK, and 16-QAM respectively. To put these results into perspective, Fig. 5 shows the packet error rate (PER) with SNR according to the model that is implemented in both VEINS and ns3 [16]. Based on the high SNR values for all modulation schemes, the PER will be zero.

In order to explain the packet loss that causes the increase of the UD in Fig. 4. Fig. 6 shows the CCDF of the signal-

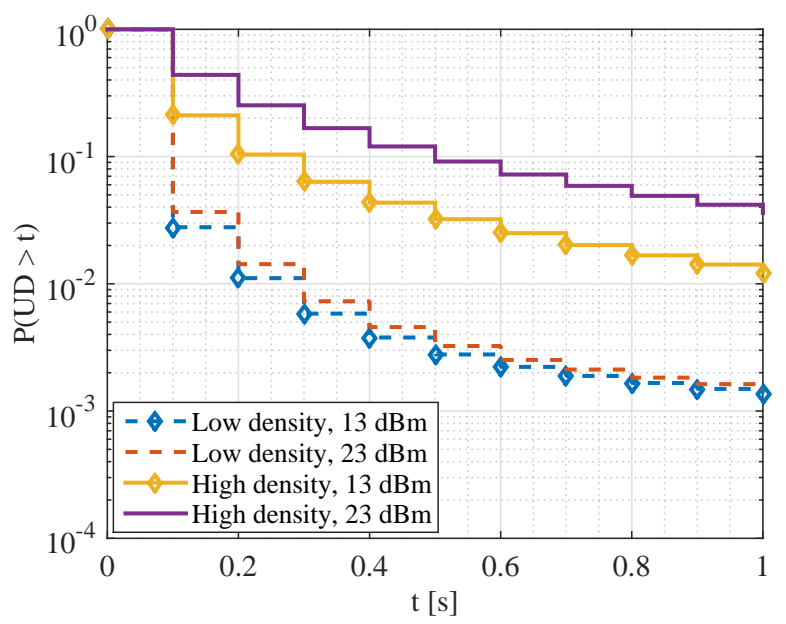

Fig. 3: CCDF of the UD in different traffic densities and transmission power at a closed intersection and a data rate of 6 Mbps.

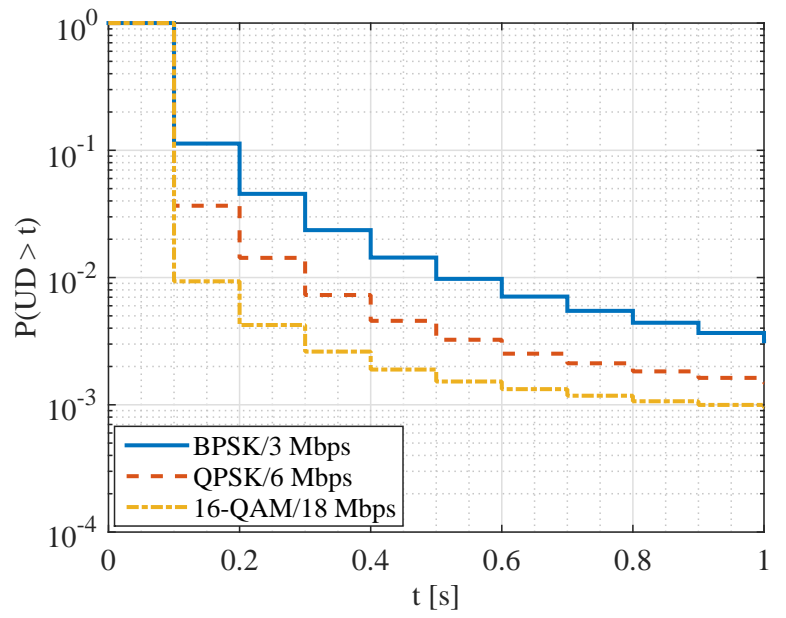

Fig. 4: CCDF of the UD at closed intersection, low traffic density and transmission power $23 \mathrm{dBm}$ for different data rates.

to-noise-and-interference (SNIR) ratio. It can be clearly seen that higher order modulation reduces interference as the packet length is shorter because of the higher spectral efficiency. This interference results from other vehicles within the communication range causing packet collisions. Thus, the only cause of packet loss is the interference and increasing the data rate reduces the UD in Fig. 4.

TABLE IV: Channel busy ratio at the intersection.

\begin{tabular}{llll}
\hline Density & 3 Mbps & 6 Mbps & 18 Mbps \\
\hline Low & 0.75 & 0.47 & 0.12 \\
\hline High & 0.95 & 0.89 & 0.50 \\
\hline
\end{tabular}

This is additionally supported by the results of the channel busy ratio (CBR), as shown in Table IV. The CBR decreases 


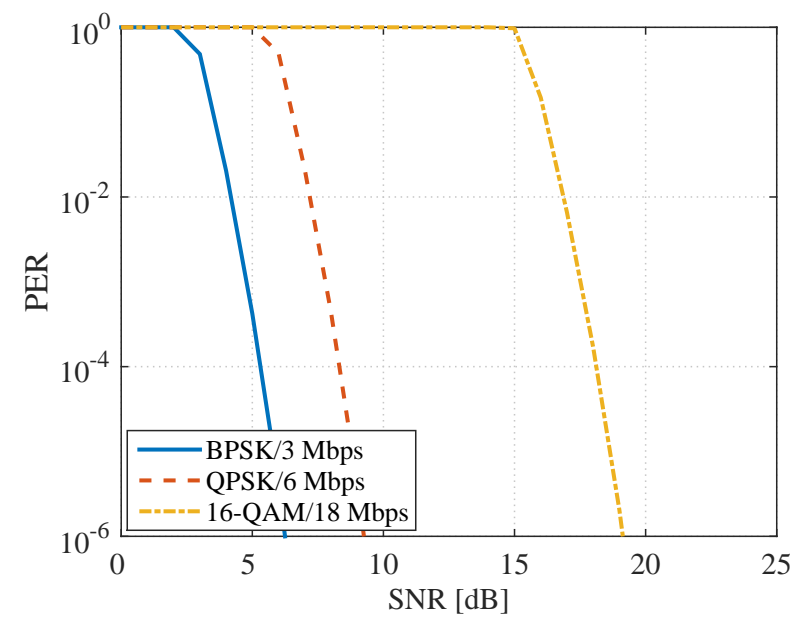

Fig. 5: Packet error rate for different data rates according to VEINS model.

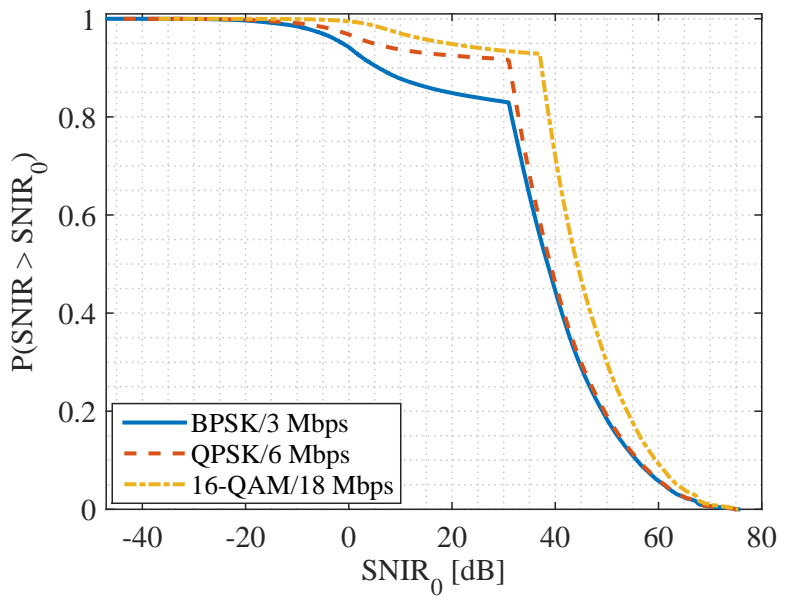

Fig. 6: CCDF of signal-to-noise-and-interference ratio at closed intersection, low traffic density and transmission power $23 \mathrm{dBm}$ for different data rates.

with increasing the data rate. This applies for both high and low density scenarios.

\section{CONCLUSiON}

In this work we evaluated the performance of the ITSG5 802.11p based V2V communication for a cooperative collision avoidance system at urban intersection during the detection phase. We have addressed the problem of shadowing from buildings and the interference from the surrounding vehicles at the intersection via simulations of the communication in realistic intersection scenarios. Furthermore, we have studied the effect of transmission power and data rate on the performance of the communication system. Our main metric was the UD which considers the up-to-dateness of a vehicle state information. We have shown in simulations that buildings at intersections can reduce interference by strongly attenuating the signals. Additionally, we found that the higher the traffic density the higher the UD. Further, our results indicate that the main reason for packet loss is the interference. Therefore, for collision avoidance scenarios, where especially short range communication is of highest importance, we found that increasing the data rate by using higher order modulation will increase robustness and achieve a better reliability for the application.

\section{REFERENCES}

[1] Statistisches Bundesamt,"Verkehrsunfälle." Germany 2016.

[2] Zhou, Jun, and Sebastian Hoyos. "Asynchronous compressive multichannel radar for interference-robust vehicle collision avoidance systems." Wireless and Microwave Circuits and Systems (WMCS), 2014 Texas Symposium on. IEEE, 2014.

[3] Velupillai, Sankaranarayanan, and Levent Guvenc. "Laser scanners for driver-assistance systems in intelligent vehicles [applications of control]." IEEE Control Systems 29.2 (2009): 17-19.

[4] Liu, Xin, Zhenping Sun, and Hangen He. "On-road vehicle detection fusing radar and vision.”Vehicular Electronics and Safety (ICVES), 2011 IEEE International Conference on. IEEE, 2011

[5] Najm, Wassim G., Jonathan Koopmann, John D. Smith, and John Brewer. "Frequency of target crashes for intellidrive safety systems." No. HS-811 381. 2010.

[6] Mangel, Thomas, Oliver Klemp, and Hannes Hartenstein. "5.9 GHz intervehicle communication at intersections: a validated non-line-of-sight pathloss and fading model.” EURASIP Journal on Wireless Communications and Networking 2011.1: 1-11.

[7] Mahler, Kim, Panagiotis Paschalidis, Mike Wisotzki, Andreas Kortke, and Wilhelm Keusgen. "Evaluation of vehicular communication performance at street intersections.'In Vehicular Technology Conference (VTC Fall), 2014 IEEE 80th, pp. 1-5. IEEE, 2014.

[8] Sommer, Christoph, Stefan Joerer, Michele Segata, Ozan K. Tonguz, Renato Lo Cigno, and Falko Dressler. "How shadowing hurts vehicular communications and how dynamic beaconing can help." IEEE Transactions on Mobile Computing 14, no. 7 (2015): 1411-1421.

[9] Zinchenko, Tetiana, Hugues Tchouankem, and Lars Wolf. "Reliability of vehicle-to-vehicle communication at urban intersections." Communication Technologies for Vehicles (Nets4Cars-Fall), 2014 7th International Workshop on. IEEE, 2014

[10] Le, Hong Quy and Rashdan, Ibrahim and Sand, Stephan (2016) "Communication Protocol for Platoon of Electric Vehicles in Mixed Traffic Scenarios." VENITS 2016, 2nd International Workshop on Vehicular Networking and Intelligent Transportation Systems, 04 Sep 2016, Valencia, Spain

[11] Bai, Fan, and Hariharan Krishnan. "Reliability analysis of DSRC wireless communication for vehicle safety applications." In Intelligent Transportation Systems Conference, 2006. ITSC'06. IEEE, pp. 355-362. IEEE, 2006.

[12] Tchouankem, Hugues, Tetiana Zinchenko, and Henrik Schumacher. "Impact of buildings on vehicle-to-vehicle communication at urban intersections." 2015 12th Annual IEEE Consumer Communications and Networking Conference (CCNC). IEEE, 2015.

[13] ETSI TS 102 637-2 Intelligent Transport Systems (ITS); Vehicular Communications; Basic Set of Applications; Part 2 : Specification of Cooperative Awareness Basic Service," tech. rep., European Telecommunications Standards Institute (ETSI), 2011.

[14] Joerer, Stefan, Michele Segata, Bastian Bloessl, Renato Lo Cigno, Christoph Sommer, and Falko Dressler. "To crash or not to crash: Estimating its likelihood and potentials of beacon-based IVC systems." In Vehicular Networking Conference (VNC), 2012 IEEE, pp. 25-32. IEEE, 2012.

[15] Sommer, Christoph, David Eckhoff, Reinhard German, and Falko Dressler. "A computationally inexpensive empirical model of IEEE 802.11 p radio shadowing in urban environments." In Wireless OnDemand Network Systems and Services (WONS), 2011 Eighth International Conference on, pp. 84-90. IEEE, 2011.

[16] Miller, L. E. "Validation of $802.11 \mathrm{a} / \mathrm{uwb}$ coexistence simulation." national institute of standards and technology (NIST), WCTG white paper (2003). 